\title{
Nuclear Magnetic Resonance Study of the Peptide FRANCESSEPAROVIC
}

\author{
John A. Karas, ${ }^{\text {A David W. Keizer, }}{ }^{\text {B }}$ and Marc-Antoine Sani ${ }^{\text {(D) B, C,D }}$ \\ A Department of Pharmacology and Therapeutics, School of Biomedical Sciences, \\ Faculty of Medicine, Dentistry and Health Sciences, The University of Melbourne, \\ Parkville, Vic. 3010, Australia. \\ ${ }^{B}$ Bio21 Institute, The University of Melbourne, Parkville, Vic. 3010, Australia. \\ ${ }^{\mathrm{C}}$ School of Chemistry, The University of Melbourne, Parkville, Vic. 3010, Australia. \\ ${ }^{\mathrm{D}}$ Corresponding author. Email: msani@unimelb.edu.au
}

\begin{abstract}
As an eminent ambassador of STEM and renowned NMR spectroscopist, Frances Separovic is an internationally famous name, but could it also be a valuable membrane-active peptide sequence? Her name has been used as an amino acid sequence (FS), successfully synthesised, oxidised, and put into contact with membrane models to investigate any serendipitous activity. The 3D structure of the cyclic FS was determined in dodecylphosphocholine (DPC) micelles by solution NMR spectroscopy. FS displayed a twisted bend separating a helical stretch and an unstructured segment. Using solid-state NMR spectroscopy, the effect of FS on 1,2-dimyristoyl-sn-glycero-3-phosphocholine (DMPC) and 1,2-dimyristoyl-sn-glycero-3-phosphoserine (DMPS) lipid bilayers was studied. FS did not strongly disturb the neutral membrane surface but likely inserted into their hydrophobic core without a strong effect on the lipid dynamics, while perturbation of the negatively charged membranes remained at the headgroup interface with a strong effect on the lipid dynamics. This study demonstrated that FS is a candidate for discovering potential future therapeutic activities.
\end{abstract}

Manuscript received: 30 July 2019.

Manuscript accepted: 4 September 2019.

Published online: 9 October 2019.

\section{Introduction}

The complex interactions between membrane-active peptides (MAP) and lipid membranes is one of the most challenging fields of structural biology. Indeed, the small size of these peptides, up to 50 amino acids, combined with a relatively transient interaction with lipids, limit the number of biophysical techniques that are able to provide high-resolution structural information on the complex lipid-peptide assembly mechanism. NMR spectroscopy is well suited to extract valuable information for such systems. Solution NMR spectroscopy, although limited by the size of the molecular system, can provide the structure of small peptides in micellar environments, while solid-state NMR spectroscopy provides a means to study peptides in large bilayered vesicle systems. The present study combined both techniques to investigate the interplay between a very particular peptide sequence and lipid membrane models.

To celebrate the contribution of the first female professor in chemistry at the University of Melbourne, we investigated a peptide with the de novo amino acid sequence FRANCESSEPAROVIC (FS). Prof. Frances Separovic has been using NMR spectroscopy to investigate several MAPs, particularly gramicidin. ${ }^{[1]}$ She provided a new understanding of how complex lipid systems modulate the membrane interaction and self-assembly of peptides. ${ }^{[2]}$ She has contributed to the development of NMR spectroscopy, especially solid-state NMR spectroscopy, ${ }^{[3]}$ in Australia with great passion and wit. In this study, we investigated the structure of FS in micelles that mimic lipid membranes and whether FS is interacting with neutral and/or anionic lipid bilayers by using Frances' favourite biophysical technique, NMR spectroscopy.

\section{Results}

FS is Bent with a Helical Hinge in Dodecylphosphocholine Micelles

The 3D structure of oxidised FS peptide in $d_{38}$-dodecylphosphocholine $\left(d_{38}\right.$-DPC) micelles was determined using ${ }^{1} \mathrm{H}-{ }^{1} \mathrm{H}$ homonuclear and heteronuclear ${ }^{15} \mathrm{~N}-{ }^{1} \mathrm{H}$ and ${ }^{13} \mathrm{C}-{ }^{1} \mathrm{H}$ HSQC NMR spectroscopy. Resonance assignmeants were determined from TOCSY, COSY, and HSQC experiments to ascertain the intra-residue hydrogen resonances and thus the spin systems. The sequential walk was then performed using the NOESY spectra, starting from the well identified single ${ }^{14}$ Val residue. Residue ${ }^{1}$ Phe did not produce a backbone amide proton resonance, most likely due to fast exchange processes on the NMR time scale. The ${ }^{1} \mathrm{H}^{\mathrm{N}}$ and ${ }^{15} \mathrm{~N}$ assignments are shown in the HSQC spectrum, with the $\mathrm{NH}_{2}$ contributions of the $\mathrm{Gln}^{4}$ sidechain, the amidated Cys ${ }^{16} \mathrm{C}$-terminus, and the folded $\mathrm{Arg}^{2}$ and $\operatorname{Arg}^{12}$ (Fig. 1). Four hundred and twenty resonances were used to generate intra and inter residue distance restraints from the NOESY experiment (300 ms mixing time) and ${ }^{1} \mathrm{H},{ }^{15} \mathrm{~N}$, and ${ }^{13} \mathrm{C}$ chemical shifts were used to further restrain the dihedral angles for structure calculation. ${ }^{[4]}$ The secondary structure elements (Fig. 1) of the peptide have been calculated from the well known 

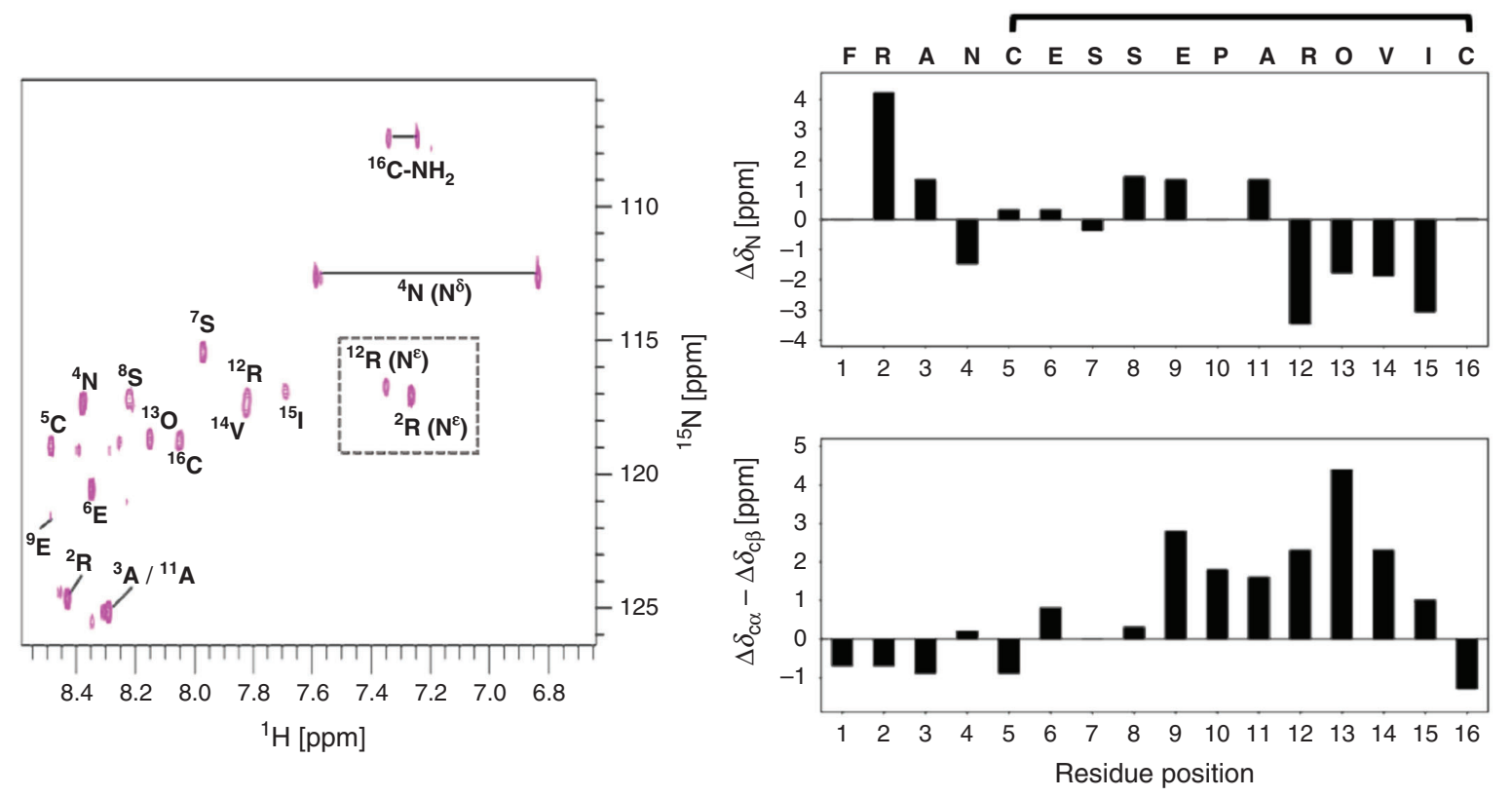

Fig. 1. (Left panel) Reconstructed ${ }^{15} \mathrm{~N}-{ }^{1} \mathrm{H}$ HSQC spectrum of natural abundance FS in $d_{38}$-DPC micelles. The peptide to detergent molar ratio was 1/100. Experiments were performed at $310 \mathrm{~K}$ with $40 \%$ non-uniform sampling. Aliased peaks for arginine residues are within the dashed box (right panels). Secondary structure analysis of FS in DPC micelles using the statistical-coil chemical shifts of Wishart et al. ${ }^{[5 b]}$

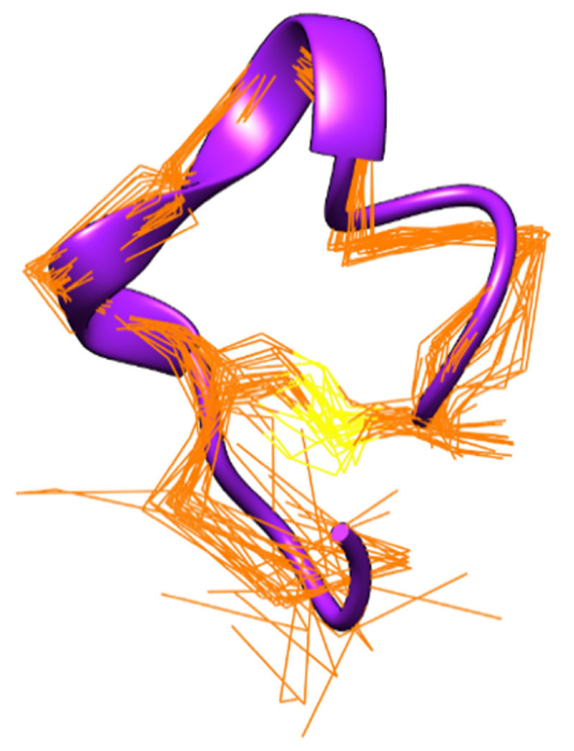

Fig. 2. Twenty of the lowest energy structures of FS in DPC micelles. The averaged structure is depicted as a purple ribbon; backbone atoms are in orange and the disulfide bond is in yellow.

empirical relations of ${ }^{13} \mathrm{C}$ chemical shifts $(\Delta \mathrm{C} \alpha-\Delta \mathrm{C} \beta)$ and $\left(\Delta \mathrm{N}^{\mathrm{H}}\right)$ to their regular secondary structure. ${ }^{[5]}$ These plots suggest a high propensity for helical structure from residues 8-15.

Ensembles of the 20 lowest energy structures refined in a water shell of FS displayed a hairpin-like structure as expected for a short cyclic peptide bearing a proline residue (Fig. 2). FS displayed a disordered region $\left(\mathrm{Phe}^{1}-\mathrm{Gln}^{4}\right)$, a twisted bend centred at $\mathrm{Glu}^{9}$, just before Pro ${ }^{10}$, and a short helical stretch (residues Ala ${ }^{11}-\mathrm{Ile}^{15}$ ). The quality and statistical analysis of the structures are listed in Table 1 and showed no dihedral violation and 13 distance violations out of the 420 restraints used. The
Table 1. Statistical information for the FS NMR structures in DPC micelles

\begin{tabular}{|c|c|}
\hline Parameter & Value \\
\hline \multicolumn{2}{|l|}{ Distance restraints } \\
\hline Total & 423 \\
\hline Intra residue & 221 \\
\hline Inter & 202 \\
\hline Sequential & 143 \\
\hline Short range (seq) & 198 \\
\hline Short range (non-seq) & 55 \\
\hline \multicolumn{2}{|c|}{ Atomic RMSD $[\AA]$ from average structure } \\
\hline Backbone (1-16) & 1.58 \\
\hline Backbone (5-16) & 0.79 \\
\hline All atoms (5-16) & 2.07 \\
\hline \multicolumn{2}{|c|}{ Violations from experimental restraints } \\
\hline NOE violations $(>0.3 \AA)$ & $13(3 \%)$ \\
\hline Average violation $[\AA]$ & 0.79 \\
\hline \multicolumn{2}{|c|}{ MolProbity statistics (on averaged structure) } \\
\hline Clashes (> $0.4 \AA / 1000$ atoms) & 16.7 \\
\hline Poor rotamers $[\%]$ & $8(1)$ \\
\hline Ramachandran outliers [\%] & $9(1)$ \\
\hline Bad bond & 0 \\
\hline Bad angle & 0 \\
\hline MolProbity score & 3.2 \\
\hline
\end{tabular}

backbone root mean square deviation (RMSD) of the ensemble for the structured $\mathrm{Cys}^{5}-\mathrm{Cys}^{16}$ region was $0.79 \AA$.

FS is Membrane Active, Inserts into a Neutral Membrane while Remaining Superficial on a Negatively Charged Membrane

The static ${ }^{31} \mathrm{P}$ spectrum of the FS-free $d_{54}$-2-dimyristoyl-snglycero-3-phosphocholine (DMPC) (d54PC) multilamellar vesicles (MLVs) acquired at $310 \mathrm{~K}$ displayed a typical axially 
(a)

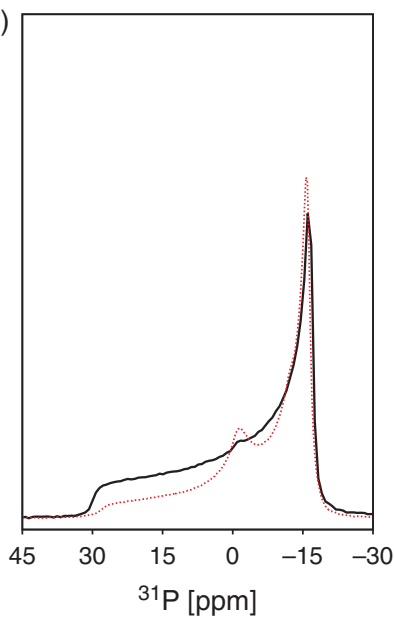

(d)

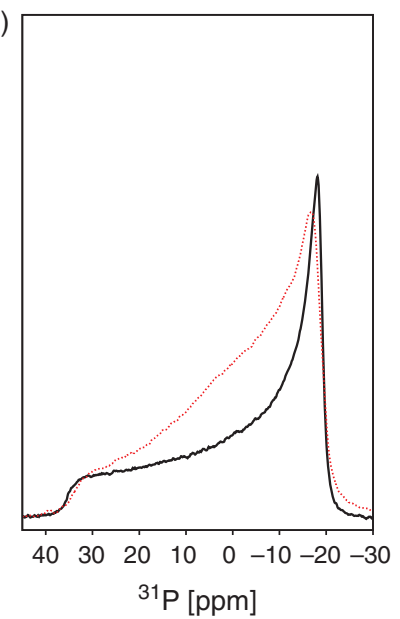

(b)

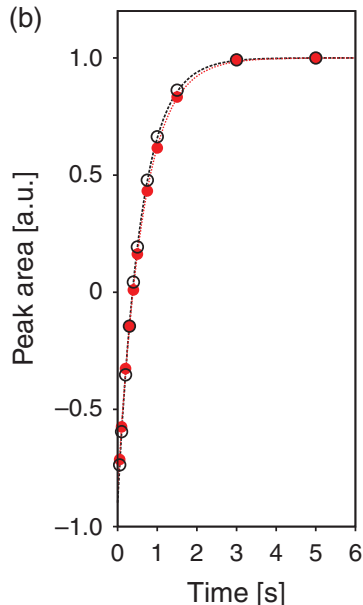

(e)

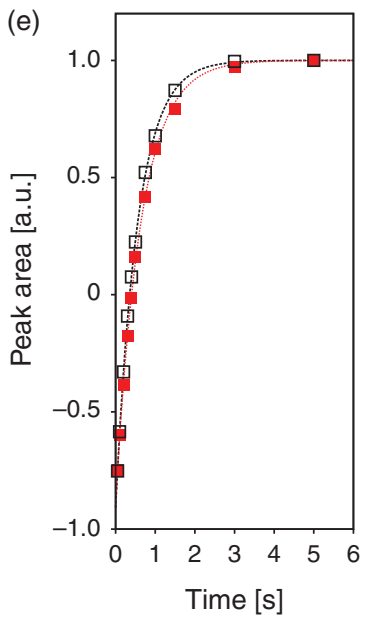

(c)

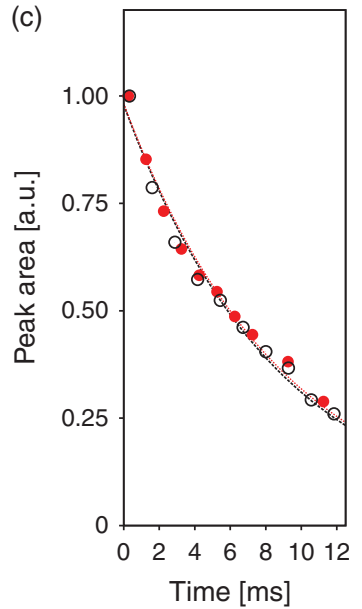

(f)

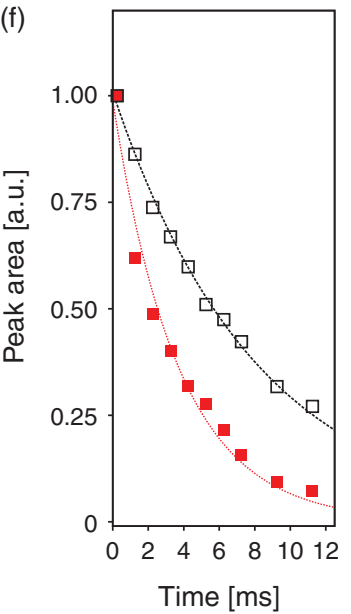

Fig. 3. Static ${ }^{31} \mathrm{P}$ solid-state NMR spectra of (a) $d_{54}$-DMPC and (d) $d_{54}$-DMPS liposomes without (solid black line) and with FS peptide at a L/P ratio of $10: 1$ (dotted red line). Experiments performed at (a) $310 \mathrm{~K}$ and (d) $313 \mathrm{~K}$. Spin-lattice $T_{1}{ }^{31} \mathrm{P}$ relaxation experiments performed on (b) $d_{54}$-DMPC liposomes without (black circles) and with FS at L/P 10: 1 (red circles) and (e) $d_{54}$-DMPS liposomes without (black squares) and with FS at L/P $10: 1$ (red squares). Spin-spin $T_{2}{ }^{31} \mathrm{P}$ relaxation experiments performed on (c) $d_{54}$-DMPC liposomes without (black circles) and with FS at L/P $10: 1$ (red circles) and (f) $d_{54}{ }^{-}$ DMPS liposomes without (black squares) and with FS at L/P 10:1 (red squares).

symmetric powder pattern with a chemical shift anisotropy (CSA) of $\sim 30.8 \mathrm{ppm}$ (Fig. 3a). The presence of FS at a lipid to peptide molar ratio $(\mathrm{L} / \mathrm{P})$ of $10: 1$ induced a small isotropic population (less than $5 \%$ ) in PC bilayers and reduced slightly the CSA by $1.8 \mathrm{ppm}$. The effect of FS on $d_{54}$-1,2-dimyristoyl-snglycero-3-phosphoserine (DMPS) (d54PS) MLVs was more pronounced, with a significant broadening of the ${ }^{31} \mathrm{P}$ lineshape, particularly near the isotropic chemical shift (Fig. 3d).

The effect of FS on the bilayer dynamics was investigated by measuring the spin-lattice $T_{1}$ and spin-spin $T_{2}$ values, which are modulated by motions at the nanosecond timescale (dominated by the rotation of the lipids along their long axis) and by motions at the millisecond to microsecond timescale (controlled by the collective wobbling motions of the lipids) respectively. ${ }^{[6]}$ At $310 \mathrm{~K}$, the $T_{1}$ and $T_{2}$ values of the MLVs with and without FS were not significantly different, $\sim 0.60 \mathrm{~s}$ and $8.80 \mathrm{~ms}$ respectively (Fig. 3b, c). While FS slightly increased the spin-lattice $T_{1}$ value of d54PS bilayers from 0.55 to $0.64 \mathrm{~s}$, the spin-spin $T_{2}$ value was severely reduced from $8.10 \mathrm{~m}$ to $3.71 \mathrm{~ms}$ (Fig. 3e, f).

The deuterium quadrupolar splittings are modulated by the trans-gauche isomerisation rate of the lipid acyl chains and can be translated to the order parameter at each carbon along the chain.
Interestingly, FS induced a disordering effect on the ${ }^{2} \mathrm{H}$ quadrupolar splitting of d54PC bilayers, slightly increasing the dynamics and thus the order parameters of the bilayer hydrophobic core (Fig. 4a, b). In d54PS, the quadrupolar splitting was not different in the presence of FS, although the linewidth of the spectrum was noticeably broadened compared with the FS-free spectrum.

\section{Discussion}

MAPs are molecules under intense research focus for their ability to modulate lipid membrane biophysical properties. For instance, MAPs can disrupt lipid bilayer integrity or translocate through membranes. ${ }^{[2 \mathrm{~b}, 7]}$ Many MAPs are either derived from naturally occurring amino acid sequences or by combining these sequences with other molecules to optimise a specific therapeutic property. ${ }^{[8]}$ De novo design is another possibility whereby the peptide sequence is not mimicking a naturally occurring amino acid sequence but instead is built based on rational design inputs. In this work, we have shown for the first time that the de novo FS peptide is membrane active and thus a potential template for MAP studies. Indeed, FS peptides showed 
(a)

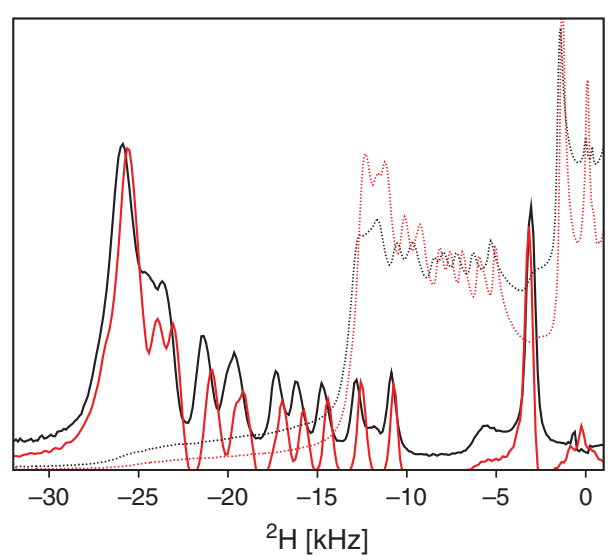

(c)

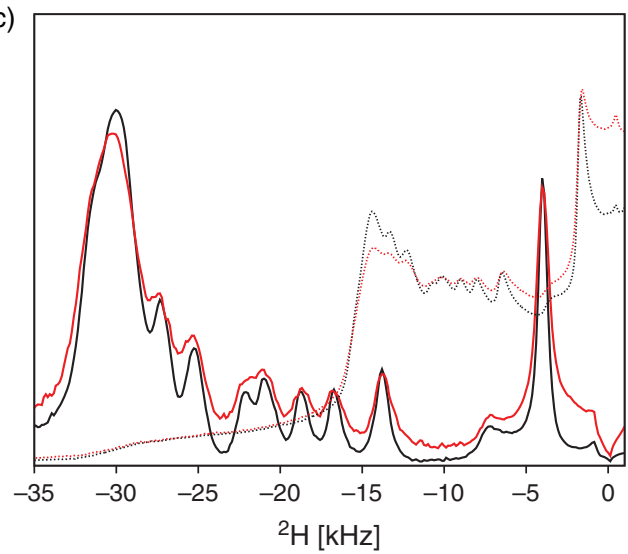

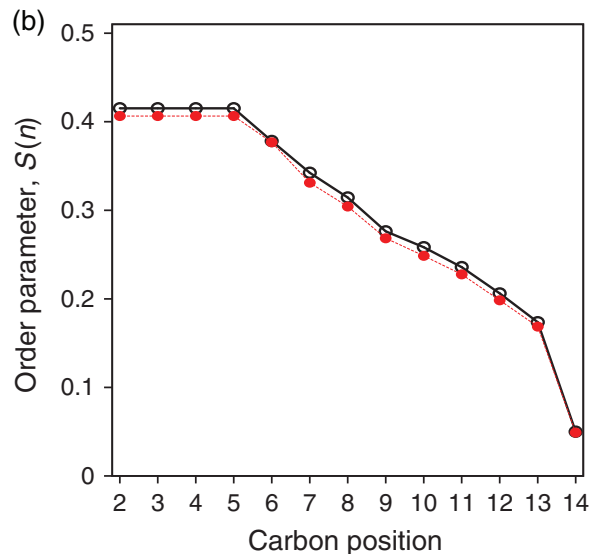

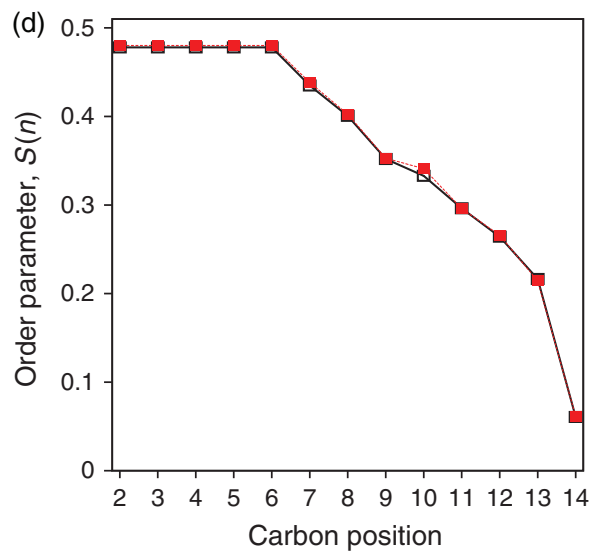

Fig. 4. Static ${ }^{2} \mathrm{H}$ solid-state NMR spectra of (a) $d_{54}$-DMPC liposomes and (c) $d_{54}$-DMPS without (dotted black line) and with FS at L/P 10 : 1 (dotted red line), and the corresponding De-Paked spectra (solid lines). Experiments performed at (a) $310 \mathrm{~K}$ and (c) $313 \mathrm{~K}$. Order parameter profile from the $0^{\circ}$ de-Paked spectra quadrupolar splittings of (b) $d_{54}$-DMPC liposomes without (black circles) and with FS at L/P 10:1 (red circles) and (d) $d_{54}$-DMPC liposomes without (black squares) and with FS at L/P 10:1 (red squares).

interesting interactions with lipid vesicles, similar to antimicrobial peptides such as maculatin ${ }^{[2 \mathrm{c}, 9]}$ and gramicidin. ${ }^{[10]}$ It is possible that FS could have some serendipitous antimicrobial and anticancer properties, although this should be further investigated with in vitro and in vivo bioassays. Furthermore, in situ solid-state NMR experiments monitoring the response of bacterial membranes under FS stress could be of interest. ${ }^{[2 \mathrm{~d}, 11]}$ It is noteworthy that cyclic FS may be resistant to in situ degradation, as often reported for similar membrane-active cyclic peptides. ${ }^{[12]}$ FS could also be utilised as a molecular scaffold for combinatorial libraries, with the randomised residues contained within the macrocycle. This would also enable other pharmacologically useful moieties to be grafted onto the N-terminal tail, such as cell-penetrating peptide motifs. Since the structure of FS in DPC micelles has been determined, molecular dynamic studies could also be undertaken which may provide further atomic details as to how FS interacts with lipid membranes (Fig. 5). We are looking forward to continue working with FS and Frances, and maybe combining the two may provide new understanding of how MAPs work in cells?

\section{Experimental}

\section{Materials}

Peptide synthesis was performed on a CEM Liberty microwave peptide synthesiser (CEM Corporation, NC, USA). All reagents were purchased from commercial sources and used without

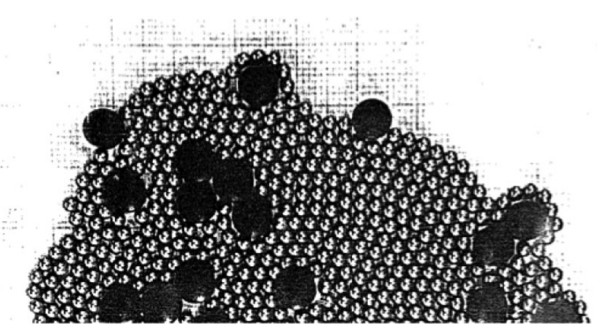

Fig. 5. Example of a non-computerised molecular dynamic simulation performed by Prof. Frances Separovic some time ago, using Brownian motions and population quantification by hand methods: lipids (small beads) and peptides (black large beads) were randomly distributed on a tray, handshaken vigorously, and snapshots of ensemble states recorded on a photographic film.

further purification. Fmoc-Rink amide resin (sub. $=0.43 \mathrm{mmol} \mathrm{g}^{-1}$ ) was sourced from Chempep (FL, USA). All standard Fmoc amino acids were purchased from CEM Corporation (NC, USA). 2-(6-Chloro- $1 H$-benzotriazole-1-yl)1,1,3,3-tetramethylaminium hexafluorophosphate (HCTU) was purchased from Iris Biotech $\mathrm{GmbH}$ (Marktredwitz, Germany). Triisopropylsilane (TIPS), 2,2'-(ethylenedioxy)diethanethiol (DODT), and 2,2'-dipyridinedisulfide (DPDS) were sourced from Sigma-Aldrich (Castle Hill, Australia). Piperidine, diisopropylethylamine (DIEA), dimethylformamide (DMF), 
dichloromethane (DCM), diethyl ether, and acetonitrile were purchased from Merck Millipore (Bayswater, Australia). Trifluoroacetic acid (TFA) was purchased from Auspep Pty Ltd (Tullamarine, Australia).

\section{Peptide Synthesis}

The FS peptide was assembled on Rink amide resin on a $0.1 \mathrm{mmol}$ scale via automated microwave-assisted peptide synthesis. The resin was then washed with DCM, followed by cleavage from the solid support and global deprotection with $10 \mathrm{~mL}$ of a TFA/DODT/TIPS/ $\mathrm{H}_{2} \mathrm{O}(95 / 2 / 2 / 1 \%)$ solution. After $2 \mathrm{~h}$, the mixture was filtered into a $50 \mathrm{~mL}$ tube and the volume of the TFA solution was reduced to $\sim 2 \mathrm{~mL}$ via $\mathrm{N}_{2}$ aspiration, followed by precipitation of the peptide with diethyl ether $(30 \mathrm{~mL})$. The tube was centrifuged, and the supernatant discarded. This process was repeated, followed by air drying of the white powder. The crude mass recovered was $205 \mathrm{mg}$ and the major product detected via liquid chromatography-mass spectrometry (LC-MS) was the linear precursor. One hundred milligrams of the crude material was then dissolved in $50 \mathrm{~mL}$ of $25 \%(\mathrm{v} / \mathrm{v})$ acetonitrile in water, followed by the addition of DPDS (1 equiv.). The reaction was stirred for $30 \mathrm{~min}$ and monitored via LC-MS. Once the cyclisation was complete, the solution was filtered and pumped directly onto a HPLC column and purified as described below. The clean fractions were pooled and lyophilised, to afford $22 \mathrm{mg}$ of pure peptide at $>95 \%$ purity.

\section{Analysis}

All analyses were conducted on an Agilent 6120 Quadrupole LC/MS with an Agilent 1260 infinity II high performance liquid chromatography (HPLC) system (Agilent Technologies Australia, Mulgrave, Australia). The mobile phases were: $0.05 \%(\mathrm{v} / \mathrm{v})$ TFA in water (buffer A) and $0.05 \%(\mathrm{v} / \mathrm{v})$ TFA in acetonitrile (buffer B). Column: Agilent Poroshell 120, EC-C8, $3 \times 50 \mathrm{~mm}, 2.7 \mu \mathrm{m}$ HPLC column. Gradient: $0-100 \%$ buffer B

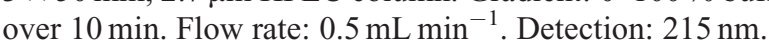

\section{Preparative HPLC}

All purification was carried out on an Agilent 1200 Series HPLC (Agilent Technologies Australia, Mulgrave, Australia). The mobile phases were: $0.1 \%(\mathrm{v} / \mathrm{v})$ TFA in water (buffer A) and $0.1 \%(\mathrm{v} / \mathrm{v})$ TFA in acetonitrile (buffer B). Column: Agilent Pursuit XRs $100 \AA$ C18, $21.2 \times 150 \mathrm{~mm}, 5 \mu \mathrm{m}$. Gradient:

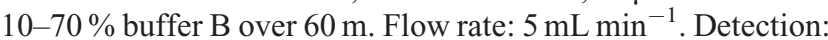
$230 \mathrm{~nm}$. Trifluoroacetate counter ions were subsequently removed by treatment with $10 \mathrm{mM} \mathrm{HCl}$ buffer and lyophilisation. ${ }^{[13]}$

\section{Solution NMR Experiments}

NMR samples were prepared by dissolving the dry peptide in $150 \mathrm{mM} d_{38}$-DPC $(20 \mathrm{mM}$ phosphate, $\mathrm{pH} 5,50 \mathrm{mM} \mathrm{KCl}$, $0.05 \mathrm{mM}$ DSS, $10 \% \mathrm{v} / \mathrm{v} \mathrm{D}_{2} \mathrm{O}$ ) to reach a final peptide concentration of $1.5 \mathrm{mM}$. NMR spectra were obtained at $310 \mathrm{~K}$ on a $800 \mathrm{MHz}$ Bruker Avance II equipped with a $5 \mathrm{~mm}$ TXI cryoprobe (Rheinstetten, Germany). ${ }^{1} \mathrm{H}$ chemical shifts were referenced to DSS (4,4-dimethyl-4-silapentane-1-sulfonic acid) at $0 \mathrm{ppm}$. The ${ }^{13} \mathrm{C}$ and ${ }^{15} \mathrm{~N}$ chemical shifts were indirectly referenced to DSS and since the statistical-coil chemical shifts of ornithine are not available, the corresponding values for lysine were adopted. Data were processed in Topspin 3.2 (Bruker) and analysed using the CCPNmr Analysis program. ${ }^{[14]}$ Backbone and side chains were assigned using ${ }^{1} \mathrm{H}$ homonuclear COSY, TOCSY (mixing time $\left.\tau_{\text {mix }}=80 \mathrm{~ms}\right), \operatorname{NOESY}\left(\tau_{\text {mix }}=100,200\right.$, $300 \mathrm{~ms}$ ), and ${ }^{13} \mathrm{C}-{ }^{1} \mathrm{H}$ and ${ }^{15} \mathrm{~N}-{ }^{1} \mathrm{H}$ HSQC experiments. The latter was acquired with non-uniform sampling $(40 \%)$ and the data was reconstructed using the compressed sensing algorithm in $q M D D .^{[15]}$

\section{Structure Calculations}

The NOESY cross-peak assignments were subsequently used to generate distance restraints for the structure determination. The NOE distance restraints were supplemented with dihedral angle restraints predicted with DANGLE from $\mathrm{H}_{\alpha}, \mathrm{H}_{\mathrm{N}}, \mathrm{C}_{\alpha}, \mathrm{C}_{\beta}$, and $\mathrm{N}_{\mathrm{H}}$ chemical shifts. ${ }^{[4]}$ A standard CNS 1.1-based protocol was employed using the ARIA 2.2 interface. ${ }^{[16]}$ The 20 lowest energy structures were refined in a water shell and evaluated with MolProbity. ${ }^{[17]}$

\section{Solid-State NMR Spectroscopy}

$d_{54}$-DMPC or $d_{54}$-DMPS and FS were co-solubilised in methanol/chloroform at a lipid to peptide (L/P) molar ratio of 10 to 1 . The organic solvent was evaporated under nitrogen atmosphere and under high vacuum overnight. The dry film was then resuspended with a filtered HEPES buffer $(20 \mathrm{mM}$ HEPES, $100 \mathrm{mM} \mathrm{NaCl}, \mathrm{pH} 7.4$ ) at a hydration level of $65 \mathrm{wt}-\%$. Three freeze-thaw cycles with vortexing were applied before packing the pellet into a $3.2 \mathrm{~mm}$ NMR rotor.

\section{${ }^{31}$ P Solid-State NMR Spectroscopy}

The static ${ }^{31} \mathrm{P}$ solid-state NMR experiments were performed on a Varian Vnmrs $600 \mathrm{MHz}$ spectrometer (Palo Alto, CA, USA) with a $3.2 \mathrm{~mm}$ HXY MAS probe. The Hahn echo pulse sequence was used with $55.5 \mathrm{kHz}{ }^{31} \mathrm{P}$ excitation, $65.7 \mathrm{kHz}$ SPINAL64 decoupling, and an echo delay of $32 \mathrm{~ms}$. The spectra were recorded with a $125 \mathrm{kHz}$ spectroscopic window, acquisition time of $8.192 \mathrm{~ms}$, and typically $10 \mathrm{k}$ transients were accumulated. The FIDs were processed using $4 \mathrm{k}$ zero filling and a $50 \mathrm{~Hz}$ linebroadening using NMRPipe. ${ }^{[18]}$

The ${ }^{31} \mathrm{P} T_{1}$ experiments were performed at $8 \mathrm{kHz}$ magic angle spinning speed using an inversion-recovery pulse sequence with $55.5 \mathrm{kHz}{ }^{31} \mathrm{P}$ excitation and $65.7 \mathrm{kHz}$ SPINAL64 decoupling. The spectra were recorded with a $125 \mathrm{kHz}$ spectroscopic window, acquisition time of $16.384 \mathrm{~ms}$, and typically 128 transients were accumulated. The FIDs were processed using $8 \mathrm{k}$ zero filling and a $50 \mathrm{~Hz}$ line broadening. The spectra were integrated, and the areas were plotted against the time delays. A monoexponential decay was used to fit the data in gnuplot (open source, http://www.gnuplot.info).

The ${ }^{31} \mathrm{P} T_{2}$ experiments were performed at $8 \mathrm{kHz}$ magic angle spinning speed using rotor-synchronised Hahn-echo pulse sequences with $55.5 \mathrm{kHz}{ }^{31} \mathrm{P}$ excitation and $65.7 \mathrm{kHz}$ SPINAL64 decoupling. The spectra were recorded with a $125 \mathrm{kHz}$ spectroscopic window, acquisition time of $16.384 \mathrm{~ms}$, and typically 128 transients were accumulated. The FIDs were processed using $8 \mathrm{k}$ zero filling and a $50 \mathrm{~Hz}$ line broadening. The spectra were integrated and the areas were plotted against the echo delays. A mono exponential decay was used to fit the data in gnuplot.

\section{${ }^{2} \mathrm{H}$ Solid-State NMR Spectroscopy}

The static ${ }^{2} \mathrm{H}$ solid-state NMR experiments were performed on a Varian Vnmrs $600 \mathrm{MHz}$ spectrometer (Palo Alto, CA, USA) with a $3.2 \mathrm{~mm}$ HXY MAS probe. The solid echo pulse 
sequence was used with $45.5 \mathrm{kHz}{ }^{2} \mathrm{H}$ excitation and an echo delay of $26 \mathrm{~ms}$. The spectra were recorded with a $500 \mathrm{kHz}$ spectroscopic window, acquisition time of $8.192 \mathrm{~ms}$, and typically $65 \mathrm{k}$ transients were accumulated. The FID were processed using $4 \mathrm{k}$ zero filling and a $50 \mathrm{~Hz}$ linebroadening. The de-Paked spectra were obtained using a NMRPipe built-in macro. ${ }^{[19]}$ The order parameters $S(n)$ were calculated from the de-Paked quadrupolar splittings using Eqn 1:

$$
\Delta v_{i}=\frac{3}{4} \frac{\left(e^{2} q Q\right)}{h} S(n)
$$

where the quadrupolar coupling constant $\frac{\left(e^{2} q Q\right)}{h}=167 \mathrm{kHz}$ and $\Delta v_{i}$ are the quadrupolar splitting at each carbon site.

\section{Conflicts of Interest}

The authors declare no conflicts of interest.

\section{Acknowledgements}

This research was funded by the Australian Research Council (ARC) Discovery Project grant DP160100959 to F.S. and LIEF grant LE160100120 to F.S., D.K. Keizer, and M.-A.S. Access to the NMR spectrometers was supported through a collaboration with the Bio21 Institute, Melbourne University, Australia. The authors are grateful for having had the opportunity to work with Professor Separovic and would like to convey to her their best wishes for her future endeavours.

\section{References}

[1] (a) T. G. Meikle, A. Zabara, L. J. Waddington, F. Separovic, C. J. Drummond, C. E. Conn, Colloids Surf. B Biointerfaces 2017, 152, 143. doi:10.1016/J.COLSURFB.2017.01.004

(b) F. Separovic, S. Barker, M. Delahunty, R. Smith, Biochim. Biophys. Acta 1999, 1416, 48. doi:10.1016/S0005-2736(98)00207-7

(c) R. Smith, D. E. Thomas, F. Separovic, A. R. Atkins, B. A. Cornell, Biophys. J. 1989, 56, 307. doi:10.1016/S0006-3495(89)82677-3

(d) B. A. Cornell, F. Separovic, A. J. Baldassi, R. Smith, Biophys. J. 1988, 53, 67. doi:10.1016/S0006-3495(88)83066-2

[2] (a) M. A. Sani, F. Separovic, J. Magn. Reson. 2015, 253, 138. doi:10. 1016/J.JMR.2014.11.016

(b) M. A. Sani, F. Separovic, Acc. Chem. Res. 2016, 49, 1130. doi:10. 1021/ACS.ACCOUNTS.6B00074

(c) M. A. Sani, T. C. Whitwell, J. D. Gehman, R. M. Robins-Browne, N. Pantarat, T. J. Attard, E. C. Reynolds, N. M. O'Brien-Simpson, F. Separovic, Antimicrob. Agents Chemother. 2013, 57, 3593. doi:10. 1128/AAC.00195-13 (d) M. A. Sani, S. Zhu, V. Hofferek, F. Separovic, FASEB J. 2019, 33, fj201900931R. doi:10.1096/FJ.201900931R

[3] (a) B. A. Cornell, F. Separovic, D. E. Thomas, A. R. Atkins, R. Smith, Biochim. Biophys. Acta 1989, 985, 229. doi:10.1016/00052736(89)90368-4

(b) B. A. Cornell, F. Separovic, Eur. Biophys. J. 1988, 16, 299. doi:10. 1007/BF00254066

(c) B. A. Cornell, L. E. Weir, F. Separovic, Eur. Biophys. J. 1988, 16, 113. doi:10.1007/BF00255521

[4] M. S. Cheung, M. L. Maguire, T. J. Stevens, R. W. Broadhurst, J. Magn. Reson. 2010, 202, 223. doi:10.1016/J.JMR.2009.11.008

[5] (a) A. Bax, M. Ikura, L. E. Kay, G. Barbato, S. Spera, Ciba Found. Symp. 1991, 161, 108 .

(b) D. S. Wishart, B. D. Sykes, F. M. Richards, Biochemistry 1992, 31, 1647. doi:10.1021/BI00121A010

[6] B. A. Cornell, J. B. Davenport, F. Separovic, Biochim. Biophys. Acta 1982, 689, 337. doi:10.1016/0005-2736(82)90267-X

[7] S. Y. Zhu, M. A. Sani, F. Separovic, Pept. Sci. 2018, 110, e24061. doi:10.1002/PEP2.24061

[8] D. E. Sheard, N. M. O'Brien-Simpson, J. D. Wade, F. Separovic, Pure Appl. Chem. 2019, 91, 199. doi:10.1515/PAC-2018-0707

[9] M. A. Sani, S. T. Henriques, D. Weber, F. Separovic, J. Biol. Chem. 2015, 290, 19853. doi:10.1074/JBC.M115.643262

[10] F. Separovic, J. Gehrmann, T. Milne, B. A. Cornell, S. Y. Lin, R. Smith, Biophys. J. 1994, 67, 1495. doi:10.1016/S0006-3495(94)80623-X

[11] S. A. Overall, S. Zhu, E. Hanssen, F. Separovic, M. A. Sani, Int. J. Mol. Sci. 2019, 20, 181. doi:10.3390/IJMS20010181

[12] S. T. Henriques, Y. H. Huang, S. Chaousis, M. A. Sani, A. G. Poth, F. Separovic, D. J. Craik, Chem. Biol. 2015, 22, 1087. doi:10.1016/ J.CHEMBIOL.2015.07.012

[13] M. A. Sani, C. Loudet, G. Grobner, E. J. Dufourc, J. Pept. Sci. 2007, 13, 100. doi:10.1002/PSC.803

[14] W. F. Vranken, W. Boucher, T. J. Stevens, R. H. Fogh, A. Pajon, M. Llinas, E. L. Ulrich, J. L. Markley, J. Ionides, E. D. Laue, Proteins 2005, 59, 687. doi:10.1002/PROT.20449

[15] V. Y. Orekhov, V. A. Jaravine, Prog. Nucl. Magn. Reson. Spectrosc. 2011, 59, 271. doi:10.1016/J.PNMRS.2011.02.002

[16] W. Rieping, M. Habeck, B. Bardiaux, A. Bernard, T. E. Malliavin, M. Nilges, Bioinformatics 2007, 23, 381. doi:10.1093/BIOINFOR MATICS/BTL589

[17] V. B. Chen, W. B. Arendall, III, J. J. Headd, D. A. Keedy, R. M. Immormino, G. J. Kapral, L. W. Murray, J. S. Richardson, D. C. Richardson, Acta Crystallogr. D Biol. Crystallogr. 2010, 66, 12. doi:10.1107/S0907444909042073

[18] F. Delaglio, S. Grzesiek, G. W. Vuister, G. Zhu, J. Pfeifer, A. Bax, J. Biomol. NMR 1995, 6, 277. doi:10.1007/BF00197809

[19] M. A. Sani, D. K. Weber, F. Delaglio, F. Separovic, J. D. Gehman, PeerJ 2013, 1, e30. doi:10.7717/PEERJ.30 